\title{
SOLITARY WAVE-SERIES SOLUTIONS TO NON-LINEAR SCHRODINGER EQUATIONS
}

\author{
M. AZRAM ${ }^{1}$ AND H. ZAMAN ${ }^{2}$ \\ ${ }^{I}$ Department of Science in Engineering, \\ International Islamic University Malaysia, \\ Jalan Gombak, 53100 Kuala Lumpur, Malaysia. \\ ${ }^{2}$ Department of Mathematics, Islamia College University 25120, \\ Peshawar 25000, Pakistan. \\ azram50@hotmail.com
}

\begin{abstract}
In this paper, higher-order dispersive non-linear Schrodinger equations are studied. Their solitary wave-series solutions with continuity of the derivatives and specific discontinuity of the derivatives at the crest are presented. Furthermore, convergence of the series' solutions is also validated and discussed with the help of graphs.
\end{abstract}

ABSTRAK: Kertas ini mengkaji persamaan Schrodinger serakan taklinear turutan tinggi. Penyelesaian siri-gelombang tunggalnya dengan kamiran berterusan dan kamiran tak berterusan pada maksimum telah dibentangkan. Penumpuan penyelesaian siri juga telah diperiksa dan dibincangkan dengan bantuan graf-graf.

KEYWORDS: Schrodinger equation; solitary wave-series solution; continuity and discontinuity of derivatives at crest

\section{INTRODUCTION}

It is well-known that the Schrodinger equation plays an important role in plasma physics, quantum mechanics and wave propagation in non-linear media [1-4] . In optical fibers propagation of short pulses is governed by the nonlinear Schrodinger equation [5]. Azzouzi et al. [6] recently presented Solitary wave solutions for high dispersive cubicquintic nonlinear Schrodinger equation. Li Yao et al. [7] presented solution to nonlinear Schrodinger equation by variational principle method. The authors [8-11] discussed different ways of solutions for nonlinear Schrodinger equations.

Currently in this paper, first solitary wave technique is applied and then the homotopy analysis method (HAM) is employed for the series solution, which was introduced first by Liao [12-13]. The HAM is independent of a small or large parameter and has been applied successfully to solve nonlinear problems such as viscous flow, heat transfer, nonlinear oscillations and Thomas Fermi atom model [14-38]. Further the HAM has certain other advantages over the perturbation expansion method, the delta expansion method and the Lypanov's expansion method, that HAM allows us great freedom and flexibility: (i) to control the region of convergence; (ii) to choose the initial guess; (iii) to choose the auxiliary linear operator.

\section{MODEL SCHRODINGER EQUATIONS AND THEIR SOLITARY WAVE-SERIES SOLUTIONS}

First consider the non homogeneous linear Schrodinger equation [8] : 


$$
i \frac{\partial u(t, x)}{\partial x}+\alpha \frac{\partial^{2} u(t, x)}{\partial t^{2}}+i \mu(t, x)=F(t, x)
$$

Where $u(t, x)$ is a complex valued function. For solitary wave solution, under the transformation $\eta=x-c t, u(t, x)=a f(\eta)$ and $F(t, x)=g(\eta)$, Eq. (1) reads

$$
\text { ia } f^{\prime}(\eta)+\alpha a c^{2} f^{\prime \prime}(\eta)+i \gamma a f(\eta)=g(\eta),
$$

Where $c$ is the wave speed, $a$ the wave amplitude, and the prime denotes the derivative with respect to $\eta$. For simplicity in case of $F(t, x)$ amplitude is choosen to be 1 . Consider the case that $f(\eta)$ arrives its maximum at the origin. Obviously, $f(\eta)$ and its derivatives tend to zero as $\eta \rightarrow+\infty$. The corresponding boundary conditions of the solitary wave having discontinuity of derivatives at crest are

$$
f(0)=1, \quad f(+\infty)=0 .
$$

We are considering only two cases of forcing function $g(\eta)$ i.e., $g(\eta)=0$ and $g(\eta)=e^{-\eta}$. In order to obtain the series solution for $g(\eta)=0$, we choose

$f_{0}(\eta)=e^{-\eta}$

$\mathbf{L}(f)=f^{\prime \prime}-f$,

as initial approximation of $f$ and auxiliary linear operator $\mathrm{L}$ satisfying

$\mathrm{L}\left[C_{1} e^{-\eta}+C_{2} e^{\eta}\right]=0$.

where $C_{1}$ and $C_{2}$ are arbitrary constants.

If $p \in[0,1]$ is an embedding parameter and $\eta_{1}$ is auxiliary non zero parameter then

$(1-p) \mathbf{L}\left[\phi(\eta, p)-f_{0}(\eta)\right]=p \eta_{1} \mathbf{N}[\phi(\eta, p)]$,

subject to boundary conditions

$$
\phi(0, p)=1, \phi^{\prime}(\infty, p)=0 \text {, }
$$

where

$$
\mathbf{N}[\phi(\eta, p)]=i a \frac{\partial \phi(\eta, p)}{\partial \eta}+\alpha a c^{2} \frac{\partial^{2} \phi(\eta, p)}{\partial \eta^{2}}+i \gamma a \phi(\eta, p)
$$

and when $p=0$ and $p=1$, then

$$
\phi(\eta, 0)=f_{0}(\eta), \phi(\eta, 1)=f(\eta)
$$

As the embedding parameter $p$ increases from 0 to $1, \phi(\eta, p)$ varies (or deforms) from the initial approximation $f_{0}(\eta)$ to the solution $f(\eta)$. Using Taylor's theorem and equation (10), one obtains

$$
\phi(\eta, p)=f_{0}(\eta)+\sum_{m=1}^{\infty} f_{m}(\eta) p^{m}
$$

in which 


$$
f_{m}(\eta)=\left.\frac{1}{m !} \frac{\partial^{m} \phi(\eta, p)}{\partial p^{m}}\right|_{p=0}, \quad(m \geq 1) .
$$

Clearly, the convergence of the series (11) depends upon $\eta_{1}$. Assume that $\eta_{1}$ is selected such that the series (11) is convergent at $p=1$, then due to equation (10) we have

$$
f(\eta)=f_{0}(\eta)+\sum_{m=1}^{\infty} f_{m}(\eta)
$$

For the $m$ th order deformation problem, we differentiate equations (7) and (8) $m$-times w.r.t $p$ and then setting $p=0$ and finally dividing it by $m$ ! the $m$ th-order deformation equation for $m \geq 1$ is given by

$$
\begin{aligned}
& \mathrm{L}\left[f_{m}(\eta)-\chi_{m} f_{m-1}(\eta)\right]=\eta_{1} \mathbf{R}_{m}(\eta), \\
& f_{m}(0)=0, \quad f_{m}(+\infty)=0,
\end{aligned}
$$

where

$$
\mathbf{R}_{m}(\eta)=i a f_{m-1}^{\prime}+\alpha a c^{2} f_{m-1}^{\prime \prime \prime}+i \gamma a f_{m-1} .
$$

To obtain the solution of above equation up to first few order of approximations, the symbolic computation software MATHEMATICA is used. The series solution up to first few order of approximations is

$$
\begin{aligned}
& \mathrm{F}[\mathrm{I} \mathrm{I}]=\mathbb{E}^{-\eta \eta}+5 \text { i } \mathrm{a} \mathbb{E}^{-\eta} \mathrm{hl} \eta+\frac{45}{8} \mathrm{a}^{2} \mathbb{E}^{-\eta} \mathrm{hl}^{2} \eta+\frac{105}{64} \mathrm{a}^{4} \mathbb{E}^{-\eta} \mathrm{hl} \mathrm{l}^{4} \eta+\frac{105}{512} \mathrm{a}^{6} \mathbb{E}^{-\eta} \mathrm{hl} \mathrm{l}^{6} \eta+ \\
& \frac{225 \mathrm{a}^{8} \mathbb{E}^{-\eta} \mathrm{hl}^{8} \eta}{32768}+\frac{7 \mathrm{a}^{10} \mathbb{E}^{-\eta} \mathrm{hl}^{10} \eta}{262144}-5 \mathrm{ac}^{2} \mathbb{E}^{-\eta \eta} \mathrm{hl} \alpha \eta+\frac{45}{2} \dot{i} \mathrm{a}^{2} \mathrm{c}^{2} \mathbb{E}^{-\eta \eta} \mathrm{hl}^{2} \alpha \eta+ \\
& \frac{45}{2} \mathrm{a}^{3} \mathrm{c}^{2} \mathbb{E}^{-\eta} \mathrm{hl} \mathrm{l}^{3} \alpha \eta+\frac{315}{64} \mathrm{a}^{5} \mathrm{c}^{2} \mathbb{E}^{-\eta} \mathrm{hl}^{5} \alpha \eta+\frac{105}{256} \mathrm{a}^{7} \mathrm{c}^{2} \mathbb{E}^{-\eta} \mathrm{hl} \mathrm{l}^{7} \alpha \eta+ \\
& \frac{225 \mathrm{a}^{9} \mathrm{c}^{2} \mathbb{E}^{-7} \mathrm{hl} \mathrm{l}^{9} \alpha \eta}{32768}-\frac{135}{8} \mathrm{a}^{2} \mathrm{c}^{4} \mathbb{E}^{-\eta} \mathrm{hl}^{2} \alpha^{2} \eta+60 \dot{\mathrm{H}} \mathrm{a}^{3} \mathrm{c}^{4} \mathbb{E}^{-\eta \eta} \mathrm{hl}^{3} \alpha^{2} \eta+ \\
& \frac{1575}{32} \mathrm{a}^{4} \mathrm{c}^{4} \mathbb{E}^{-7} \mathrm{hl}^{4} \alpha^{2} \eta+\frac{3675}{512} \mathrm{a}^{6} \mathrm{c}^{4} \mathbb{E}^{-7} \mathrm{hl}^{6} \alpha^{2} \eta+\frac{2835 \mathrm{a}^{8} \mathrm{c}^{4} \mathbb{E}^{-7} \mathrm{hl}^{8} \alpha^{2} \eta}{8192}+ \\
& \frac{495 \mathrm{a}^{10} \mathrm{c}^{4} \mathbb{E}^{-\eta} \mathrm{hl}^{10} \alpha^{2} \eta}{262144}-\frac{75}{2} \mathrm{a}^{3} \mathrm{c}^{6} \mathbb{E}^{-\eta} \mathrm{hl}^{3} \alpha^{3} \eta+105 \mathrm{il} \mathrm{a}^{4} \mathrm{c}^{6} \mathbb{E}^{-\eta} \mathrm{hl}^{4} \alpha^{3} \eta+ \\
& \frac{2205}{32} \mathrm{a}^{5} \mathrm{c}^{6} \mathbb{E}^{-7} \mathrm{hl}^{5} \alpha^{3} \eta+\frac{1575}{256} \mathrm{a}^{7} \mathrm{c}^{6} \mathbb{E}^{-7} \mathrm{hl}^{7} \alpha^{3} \eta+\frac{1155 \mathrm{a}^{9} \mathrm{c}^{6} \mathbb{E}^{-\eta} \mathrm{hl} \mathrm{l}^{9} \alpha^{3} \eta}{8192}- \\
& \frac{3675}{64} \mathrm{a}^{4} \mathrm{c}^{8} \mathbb{E}^{-\eta} \mathrm{hl} \mathrm{l}^{4} \alpha^{4} \eta+126 \dot{\mathrm{n}} \mathrm{a}^{5} \mathrm{c}^{8} \mathbb{E}^{-7} \mathrm{hl}^{5} \alpha^{4} \eta+\frac{33075}{512} \mathrm{a}^{6} \mathrm{c}^{8} \mathbb{E}^{-\eta} \mathrm{hl}^{6} \alpha^{4} \eta+\ldots \ldots
\end{aligned}
$$

when $g(\eta)=e^{-\eta}$ then solitary wave series solution having discontinuity of derivatives at crest up to first few order of approximations is 


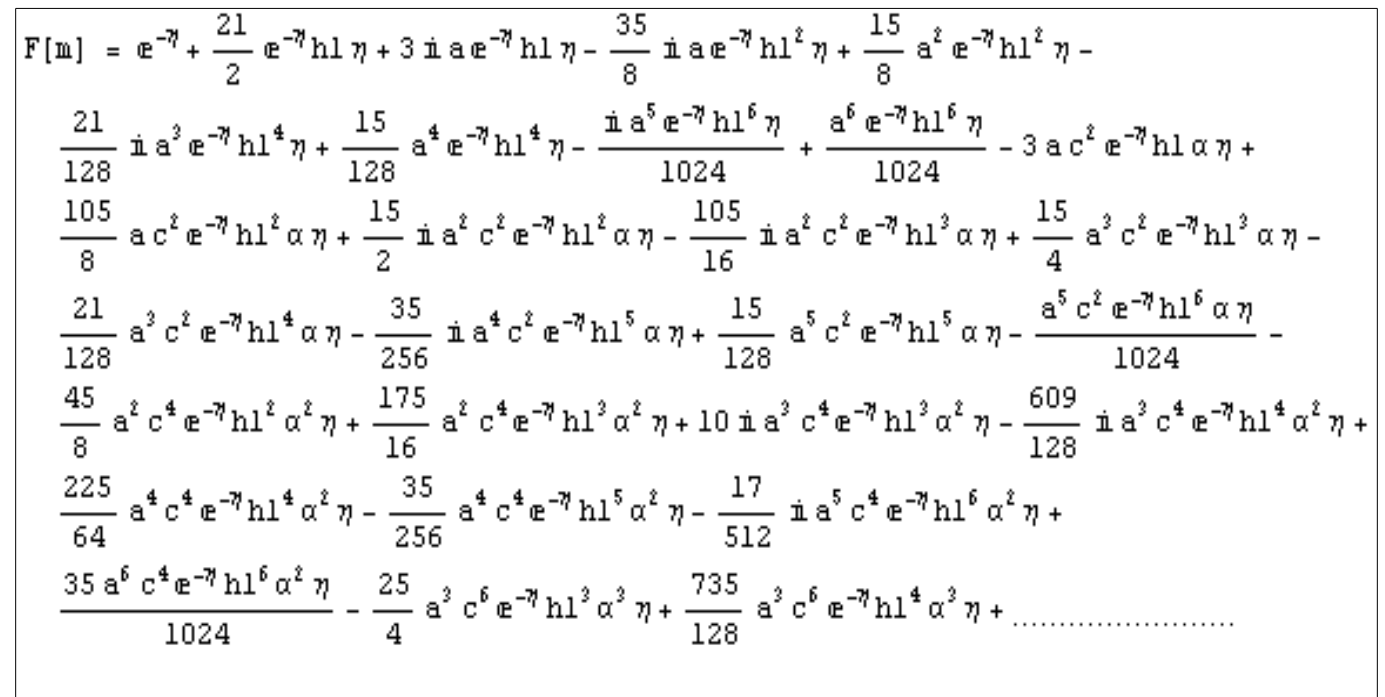

For continuity of derivatives at crest the boundary conditions are

$f(0)=1, f^{\prime}(0)=0, f(+\infty)=0$.

Taking the initial guess

$f_{0}(\eta)=2 e^{-\eta}-e^{-2 \eta}$

the solitary wave series solution, having continuity of derivatives at crest, using Eqs.

(5), (19) and (20), up to first few order of approximations are, when $g(\eta)=0$,

$$
\begin{aligned}
& F[\mathbb{i l}]=-\mathbb{E}^{-2 \eta n}+2 \mathbb{E}^{-n}+4 \dot{i} a \mathbb{E}^{-2 \eta n} \mathrm{hl}-4 \dot{1} a \mathbb{E}^{-n} \mathrm{hl}+\frac{20}{3} \mathrm{a}^{2} \mathbb{E}^{-2 \eta} \mathrm{hl}^{2}-
\end{aligned}
$$

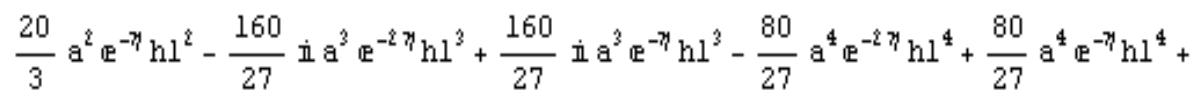

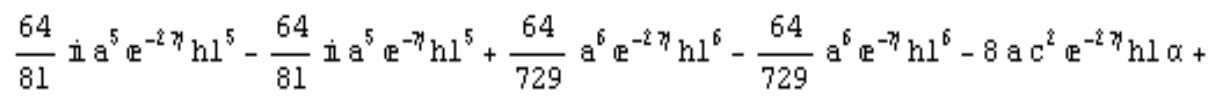

$$
\begin{aligned}
& 8 \mathrm{ac}^{2} \mathbb{E}^{-\eta} \mathrm{hl} \alpha+\frac{80}{3} \dot{\mathrm{i}} \mathrm{a}^{2} \mathrm{c}^{2} \mathbb{E}^{-2} \bar{h} \mathrm{l}^{2} \alpha-\frac{80}{3} \dot{\mathrm{i}} \mathrm{a}^{2} \mathrm{c}^{2} \mathbb{E}^{-\eta} \mathrm{hl} \mathrm{l}^{2} \alpha+\frac{320}{9} \mathrm{a}^{3} \mathrm{c}^{2} \mathbb{E}^{-2 \pi} \mathrm{hl} \mathrm{l}^{3} \alpha-
\end{aligned}
$$

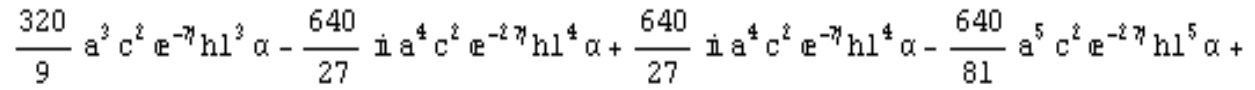

$$
\begin{aligned}
& \frac{640}{81} \mathrm{a}^{5} \mathrm{c}^{2} \mathbb{E}^{-7} \mathrm{~h} \mathrm{l}^{5} \alpha+\frac{256}{243} \dot{\mathrm{i}} \mathrm{a}^{6} \mathrm{c}^{2} \mathbb{E}^{-27} \mathrm{hl}^{6} \alpha-\frac{256}{243} \dot{\mathrm{i}} \mathrm{a}^{6} \mathrm{c}^{2} \mathbb{E}^{-7} \mathrm{hl} \mathrm{l}^{6} \alpha-\frac{80}{3} \mathrm{a}^{2} \mathrm{c}^{4} \mathbb{E}^{-27} \mathrm{hl} \mathrm{l}^{2} \alpha^{2}+ \\
& \frac{80}{3} \mathrm{a}^{2} c^{4} \mathbb{E}^{-7} h l^{2} \alpha^{2}+\frac{640}{9} \dot{i} a^{3} c^{4} \mathbb{E}^{-2} 7 h l^{3} \alpha^{2}-\frac{640}{9} \dot{i} a^{3} c^{4} \mathbb{E}^{-7 n} h l^{3} \alpha^{2}+
\end{aligned}
$$

when $g(\eta)=e^{-\eta}$, 


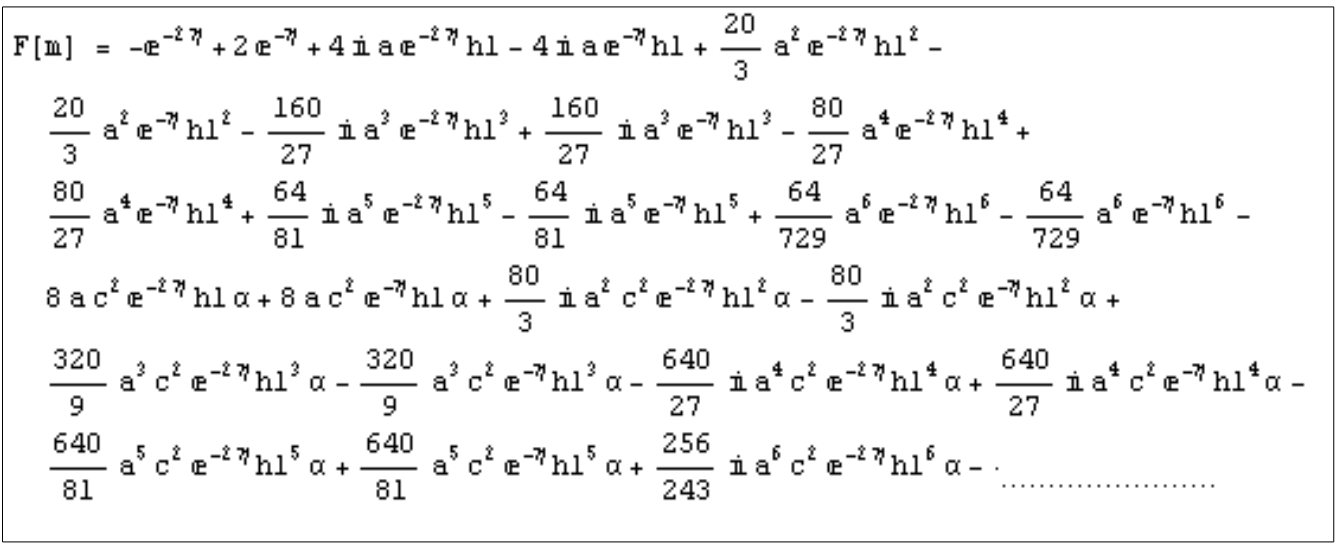

Consider the nonlinear Schrodinger equation [8] :

$$
i \frac{\partial u(t, x)}{\partial x}+\alpha \frac{\partial^{2} u(t, x)}{\partial t^{2}}+\varepsilon|u(t, x)|^{2} u(t, x)+i \xi u(t, x)=0
$$

where $u(t, x)$ is a complex valued function. For solitary wave solution Eq. (23) reduces to

$$
i a f^{\prime}(\eta)+\alpha a c^{2} f^{\prime \prime}(\eta)+\varepsilon \mid a f(\eta)^{2} a f(\eta)+i \gamma a f(\eta)=0,
$$

Under certain assumptions, as in the solution of Eq. (1), the solitary wave-series solution up to first few order of approximations at $\eta_{2}=-1 / 4$, is of the form, when discontinuity of derivatives at crest,

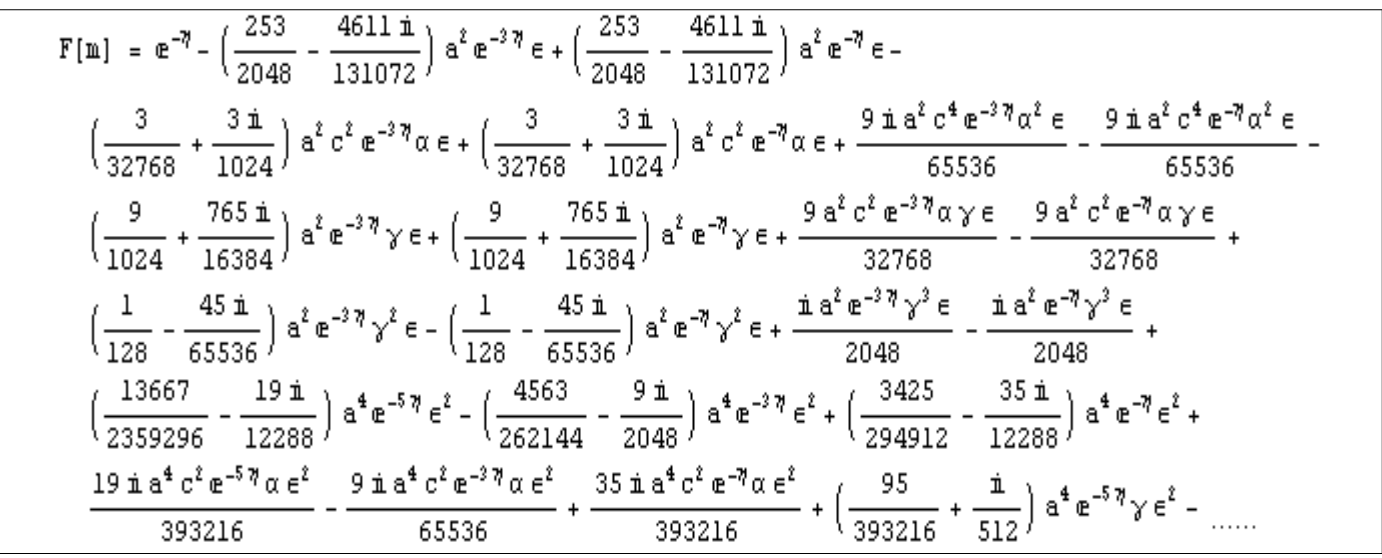

when continuity of derivatives at crest, 


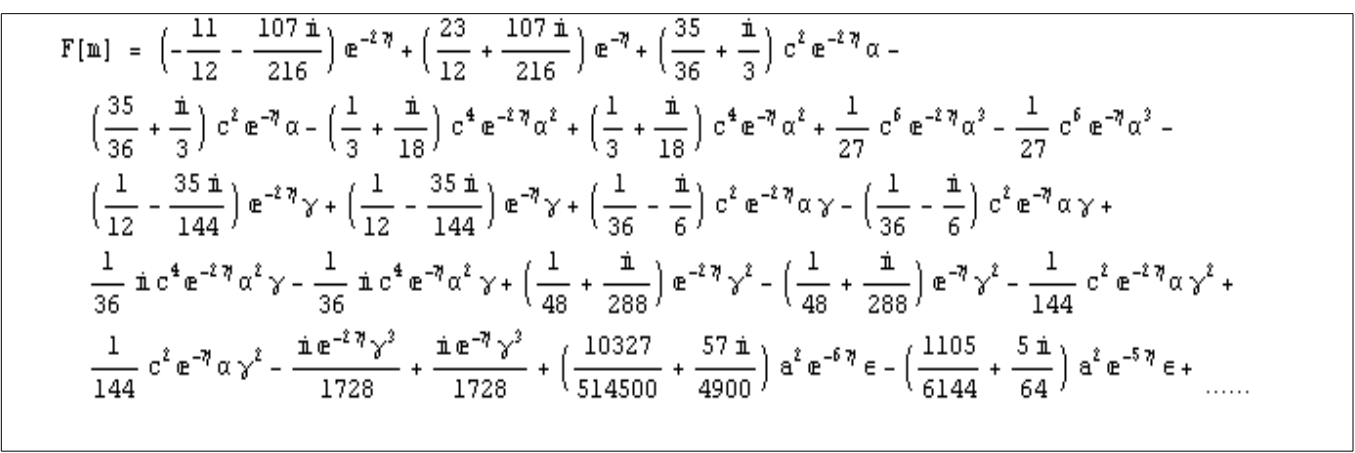

The nonlinear Schrodinger equation as considered by Xu L. and Zhang J. [10] :

$$
i \frac{\partial u(t, x)}{\partial x}-\frac{1}{2} \alpha \frac{\partial^{2} u(t, x)}{\partial t^{2}}+\beta|u(t, x)|^{2} u(t, x)+i \varepsilon \frac{\partial^{3} u(t, x)}{\partial t^{3}}+\left.i \delta u(t, x)\right|^{2} \frac{\partial u(t, x)}{\partial t}+i \mu^{2}(t, x) \frac{\partial u(t, x)}{\partial t}=0
$$

where $u(t, x)$ is a complex valued function.

Under certain assumptions, as in the solution of Eq. (1), the solitary wave-series solution up to first few order of approximations at $\eta_{3}=-1 / 4$ of Eq.(27) is of the form, when discontinuity of derivatives at crest,

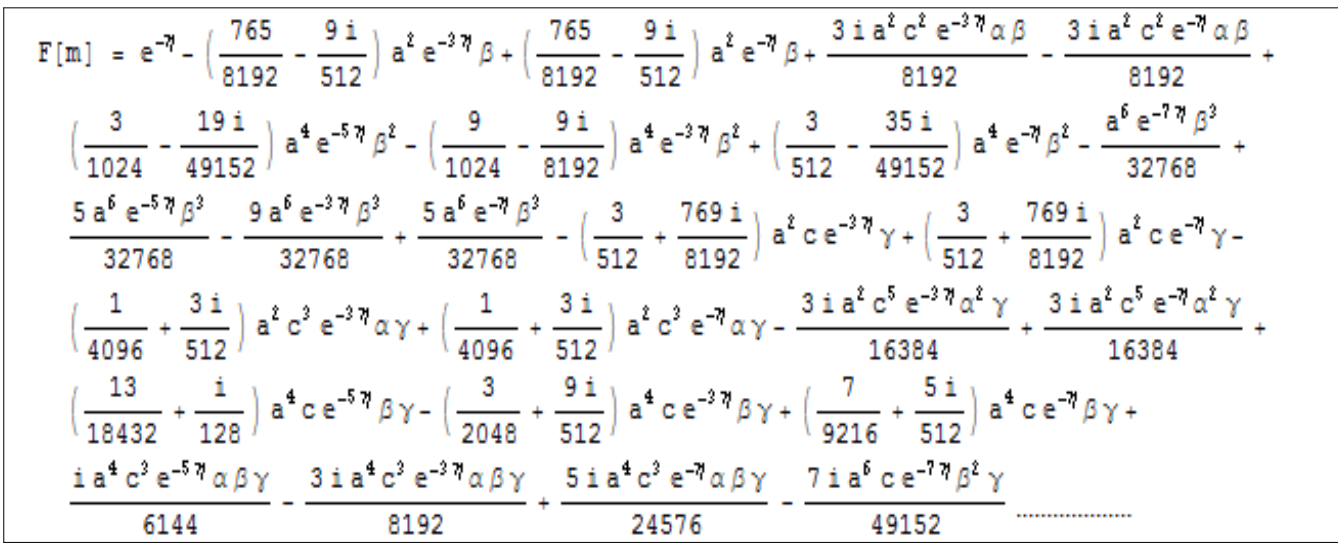

When continuity of derivatives at crest,

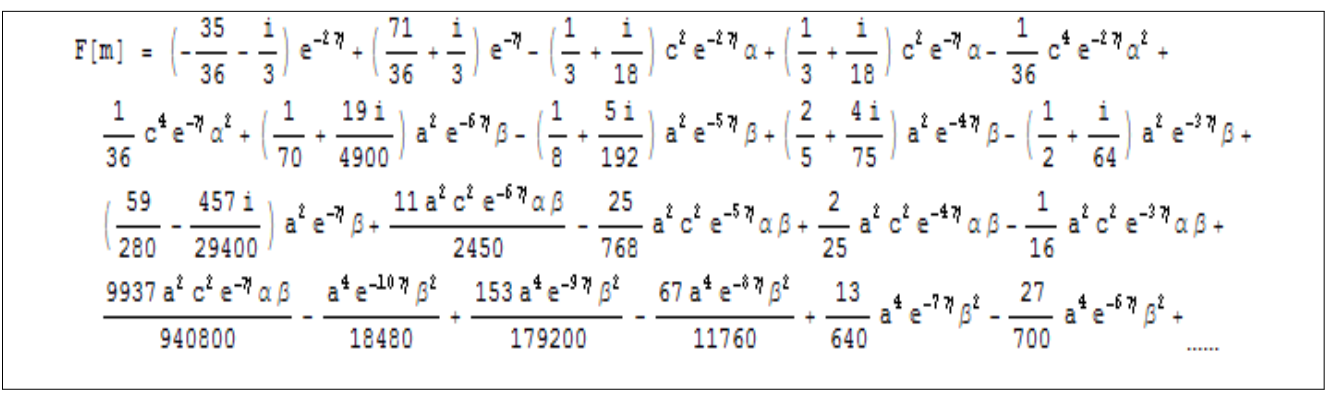


Sun et al. [11]

$i \frac{\partial \psi(t, x)}{\partial t}+\frac{\partial^{2} \psi(t, x)}{\partial x^{2}}+|\psi(t, x)|^{2} \psi(t, x)=0$,

where $\psi(t, x)$ is a complex valued function.

Under certain assumptions, as in the solution of Eq. (1), the solitary wave-series solution up to first few order of approximations at $\eta_{4}=-1$ of Eq.(30) is of the form, when discontinuity of derivatives at crest,

$$
\begin{aligned}
& F[\mathbb{i}]=-\frac{1}{512} a^{9} \mathbb{E}^{-7 \eta}+\frac{3}{64} a^{6} \mathbb{E}^{-5 \eta}+\frac{5}{512} a^{9} \mathbb{E}^{-5 \eta}+\frac{19}{768} \dot{i} a^{6} C \mathbb{E}^{-5 \eta}-\frac{3}{8} a^{3} \mathbb{E}^{-3 \eta}-\frac{9}{64} a^{6} \mathbb{E}^{-3 \eta}- \\
& \frac{9}{512} a^{9} \mathbb{E}^{-3 n}-\frac{15}{64} \dot{i} a^{3} c \mathbb{E}^{-3 n}-\frac{9}{128} \dot{i} a^{6} c \mathbb{E}^{-3 n}+\frac{3}{128} a^{3} c^{2} \mathbb{E}^{-3 n}+\mathbb{E}^{-n}+\frac{3}{8} a^{3} \mathbb{E}^{-n}+\frac{3}{32} a^{6} \mathbb{E}^{-n}+
\end{aligned}
$$

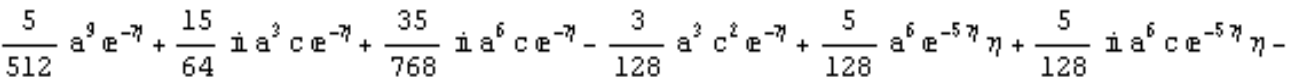

$$
\begin{aligned}
& \frac{27}{64} \mathrm{a}^{3} \mathbb{E}^{-3 \eta} \eta-\frac{9}{128} \mathrm{a}^{6} \mathbb{E}^{-3 \eta} \eta-\frac{33}{64} \dot{\mathrm{i}} \mathrm{a}^{3} c \mathbb{E}^{-3 \eta} \eta-\frac{9}{128} \dot{\mathrm{i}} \mathrm{a}^{6} c \mathbb{E}^{-3 \eta} \eta+\frac{3}{32} \mathrm{a}^{3} \mathrm{c}^{2} \mathbb{E}^{-3 \eta} \eta+\frac{11 \mathbb{E}^{-\eta} \eta}{16}+ \\
& \frac{9}{64} \mathrm{a}^{3} \mathbb{E}^{-\eta} \eta+\frac{1}{64} \mathrm{a}^{6} \mathbb{E}^{-\eta} \eta+\frac{1}{2} \dot{i} \mathrm{c} \mathbb{E}^{-\eta} \eta+\frac{11}{64} \dot{\boldsymbol{n}} \mathrm{a}^{3} \mathrm{c} \mathbb{E}^{-\eta} \eta+\frac{1}{64} \dot{\boldsymbol{i}} \mathrm{a}^{6} \mathrm{c} \mathbb{E}^{-\eta} \eta+\frac{3}{16} \mathrm{c}^{2} \mathbb{E}^{-\eta} \eta- \\
& \frac{1}{32} a^{3} c^{2} \mathbb{E}^{-n} \eta-\frac{9}{64} a^{3} \mathbb{E}^{-3} \eta \eta^{2}-\frac{9}{32} \dot{i} a^{3} c \mathbb{E}^{-3 \eta} \eta^{2}+\frac{9}{64} a^{3} c^{2} \mathbb{E}^{-3} \eta \eta^{2}+\frac{3}{16} \mathbb{E}^{-\eta} \eta^{2}+
\end{aligned}
$$

when continuity of derivatives at crest,

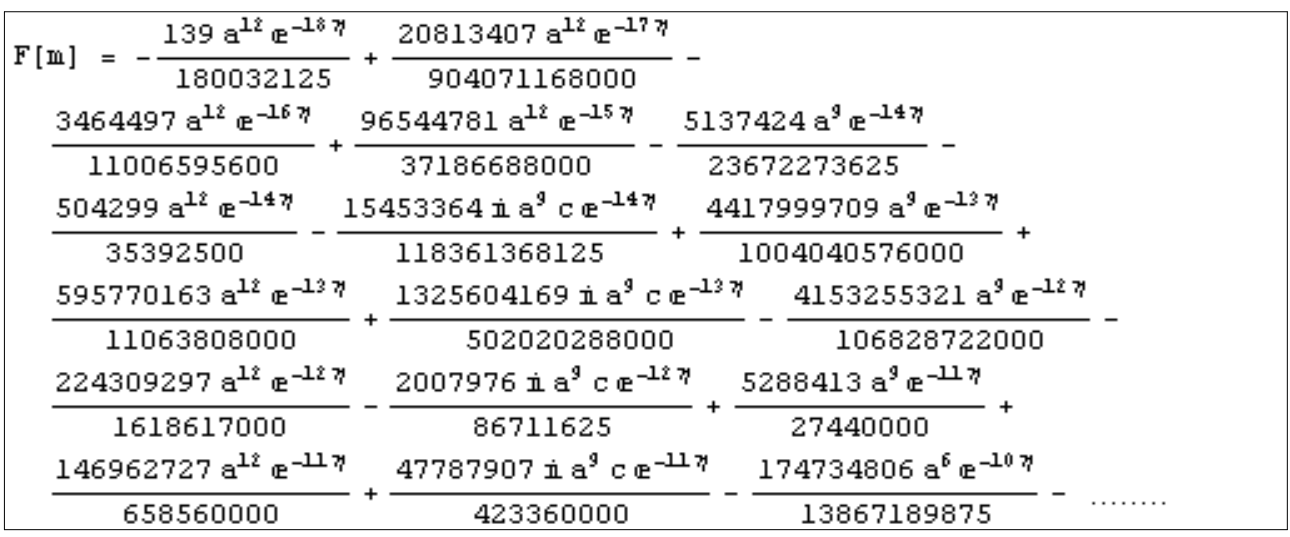

The equation considered by Azzouzi et al. [6] is

$$
\begin{aligned}
& -\frac{\partial E(t, z)}{\partial z}-i \frac{\beta_{2}}{2} \frac{\partial^{2} E(t, z)}{\partial t^{2}}+i \gamma_{1}|E(t, z)|^{2} E(t, z)+\frac{\beta_{3}}{6} \frac{\partial^{3} E(t, z)}{\partial t^{3}} \\
& +i \frac{\beta_{4}}{24} \frac{\partial^{4} E(t, z)}{\partial t^{4}}-i \gamma_{2}|E(t, z)|^{4} E(t, z)=0 .
\end{aligned}
$$

The solutions in this case at $\eta_{5}=-3 / 4$ are, when discontinuity of derivatives at crest, 


\begin{tabular}{|c|c|}
\hline$F[\mathrm{I}]=\mathbb{E}^{-\eta}+\frac{117 \mathbb{E}^{-\eta} \eta}{128}+$ & $\eta+\frac{189 \mathbb{E}^{-\eta} \eta^{2}}{1024}+\frac{9 \mathbb{E}^{-\eta} \eta^{3}}{1024}-\frac{369 \text { i } c^{2} \mathbb{E}^{-\eta} \eta \beta_{2}}{2048}-\frac{297 \text { i } c^{2} \mathbb{E}^{-\eta} \eta^{2} \beta_{2}}{2048}-$ \\
\hline 27 立 $c^{2} \mathbb{E}^{-\eta} \eta^{3} \beta_{\varepsilon}$ & 135 in $c^{6} \mathbb{E}^{-7} \eta \beta_{2}^{3}$ \\
\hline 2048 & 8192 \\
\hline 81 in $\mathrm{c}^{6} \mathbb{E}^{-7} \eta^{2} \beta_{z}^{3}$ & 9 in $\mathbb{c}^{6} \mathbb{E}^{-7} \eta^{3} \beta_{2}^{3}$ \\
\hline 8192 & 2048 \\
\hline $153 \dot{i} \mathrm{c}^{5} \mathbb{E}^{-7} \eta \beta_{2} \beta_{3}$ & 9 立 $\mathrm{c}^{5} \mathbb{E}^{-7} \eta^{3} \beta_{2} \beta_{3}$ \\
\hline 2048 & 1024 \\
\hline $99 \mathrm{c}^{7} \mathbb{E}^{-\eta n} \eta^{2} \beta_{2}^{2} \beta_{3}$ & $\beta^{2} \quad 9 \mathrm{c}^{6} \mathbb{E}^{-77} \eta^{2} \beta_{3}^{2}$ \\
\hline 8192 & 8192 \\
\hline $3 \mathrm{c}^{6} \mathbb{E}^{-7} \eta^{3} \beta_{3}^{2}$ & 39 in $c^{8} \mathbb{E}^{-\eta} \eta^{2} \beta_{2} \beta_{3}^{2}$ \\
\hline 4096 & 8192 \\
\hline
\end{tabular}

when continuity of derivatives at crest,

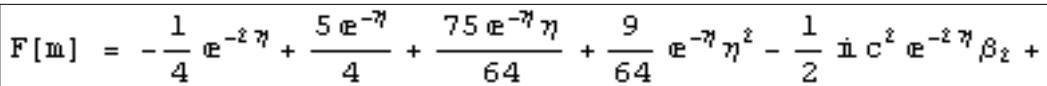

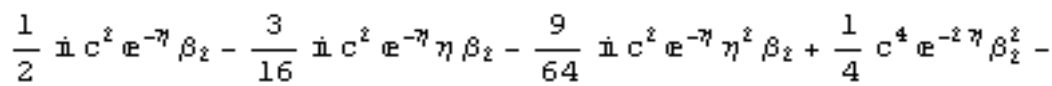

$$
\begin{aligned}
& \frac{1}{4} c^{4} \mathbb{E}^{-\eta} \beta_{2}^{2}+\frac{51}{256} c^{4} \mathbb{E}^{-\eta} \eta \beta_{2}^{2}-\frac{9}{256} c^{4} \mathbb{E}^{-7} \eta^{2} \beta_{2}^{2}+\frac{1}{3} c^{3} \mathbb{E}^{-2} \eta \beta_{3}- \\
& \frac{1}{3} c^{3} \mathbb{E}^{-7} \beta_{3}-\frac{3}{64} c^{3} \mathbb{E}^{-7} \eta \beta_{3}+\frac{3}{64} c^{3} \mathbb{E}^{-\eta} \eta^{2} \beta_{3}+\frac{1}{3} \dot{n} c^{5} \mathbb{E}^{-2} \eta \beta_{2} \beta_{3}- \\
& \frac{1}{3} \text { in } c^{5} \mathbb{E}^{-\eta} \beta_{2} \beta_{3}+\frac{3}{16} \text { in } c^{5} \mathbb{E}^{-7} \eta \beta_{2} \beta_{3}-\frac{3}{128} \text { in } c^{5} \mathbb{E}^{-\eta} \eta^{2} \beta_{2} \beta_{3}- \\
& \frac{1}{9} c^{6} \mathbb{E}^{-2 \eta \eta} \beta_{3}^{2}+\frac{1}{9} c^{6} \mathbb{E}^{-77} \beta_{3}^{2}-\frac{31}{768} c^{6} \mathbb{E}^{-7} \eta \beta_{3}^{2}+\frac{1}{256} c^{6} \mathbb{E}^{-77} \eta^{2} \beta_{3}^{2}+
\end{aligned}
$$

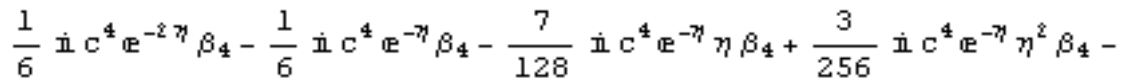

$$
\begin{aligned}
& \frac{1}{6} c^{6} \mathbb{E}^{-2} \eta_{1} \beta_{2} \beta_{4}+\frac{1}{6} c^{6} \mathbb{E}^{-\eta 7} \beta_{2} \beta_{4}-\frac{35}{512} c^{6} \mathbb{E}^{-\eta \eta} \eta \beta_{2} \beta_{4}+\frac{3}{512} c^{6} \mathbb{E}^{-7} \eta^{2} \beta_{2} \beta_{4}-
\end{aligned}
$$

\section{CONVERGENCE OF THE SERIES SOLUTIONS}

Clearly Eqs. 17, 18, 21, 22, 25, 26, 28, 29, 31, 32, 34 and 35 contains the auxiliary parameters $\eta_{1}, \eta_{2}, \eta_{3}, \eta_{4}$ and $\eta_{5}$ which gives the convergence region and rate of approximation for the homotopy analysis method. For this purpose, the $\eta$-curves are plotted for $f$ for different order of approximations. Figures 1 to 12 are plotted for the solutions given in Eqs. 17, 18, 21, 22, 25, 26, 28, 29, 31, 32, 34 and 35, respectively. It is obvious from Fig. 1 that the range for the admissible value for $\eta_{1}$ is $-0.2<\eta_{1}<0$. Figure 2 shows that the range for the admissible value for $\eta_{1}$ is $-0.52<\eta_{1}<0$. Figure 3 depicts that the range for the admissible value for $\eta_{1}$ is $-0.2<\eta_{1}<0$.

Figure 4 indicates that the range for the admissible value for $\eta_{1}$ is $-0.8<\eta_{1}<-0.4$. Figure 5 shows that the range for the admissible value for $\eta_{2}$ is $-0.3<\eta_{2}<0$. Figure 6 
describes that the range for the admissible value for $\eta_{2}$ is $-0.3<\eta_{2}<0$. Figure 7 shows that the range for the admissible value for $\eta_{3}$ is $-0.3<\eta_{3}<0$. Figure 8 indicates that the range for the admissible value for $\eta_{3}$ is $-0.3<\eta_{3}<0$. Figure 9 shows that the range for the admissible value for $\eta_{4}$ is $-1.7<\eta_{4}<-0.5$. Figure 10 shows that the range for the admissible value for $\eta_{4}$ is $-1.2<\eta_{4}<-0.7$. Figure 11 describes that the range for the admissible value for $\eta_{5}$ is $-0.7<\eta_{5}<-0.6$. Figure 12 shows that the range for the admissible value for $\eta_{5}$ is $-1.2<\eta_{5}<-0.9$. These all prescribed values of $\eta_{1}, \eta_{2}, \eta_{3}$, $\eta_{4}$ and $\eta_{5}$ in their respective intervals shows region of convergence for their respective series solutions.

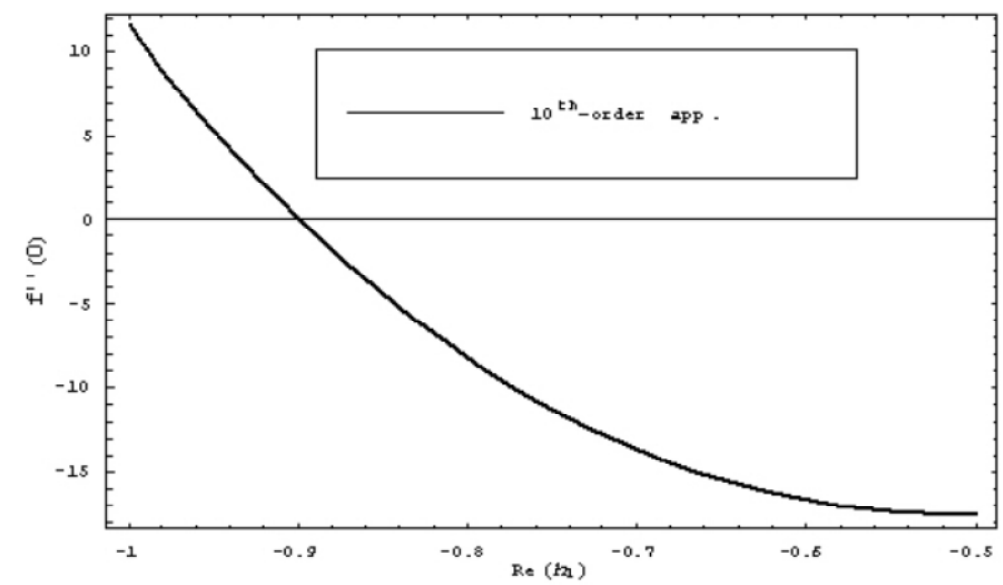

Fig. $2 \hbar_{1}$-curve for discontinuity of derivative at the crest, for the $f(\eta)$ at $\alpha=0.01, \gamma=0.01, c=0.01, a=1, g(\eta)=e^{-\eta}$.

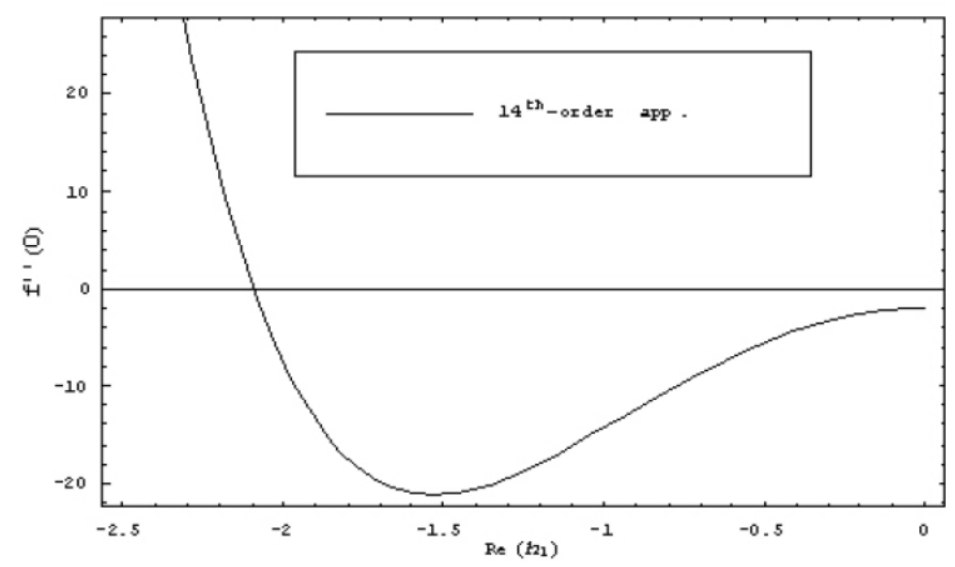

Fig. $3 \hbar_{1}$-curve for continuity of derivative at the crest, for the $f(\eta)$ at $\alpha=0.01, \gamma=0.01, c=0.01, a=1, g(\eta)=0$. 


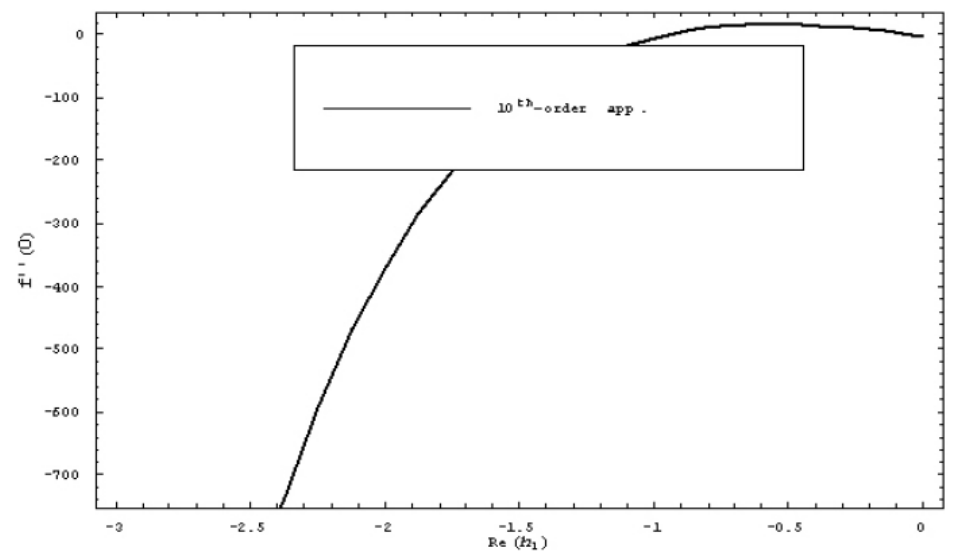

Fig. $4 \hbar_{1}$-curve for continuity of derivative at the crest, for the $f(\eta)$ at $\alpha=0.01, \gamma=0.01, c=0.01, a=1, g(\eta)=e^{-\eta}$.

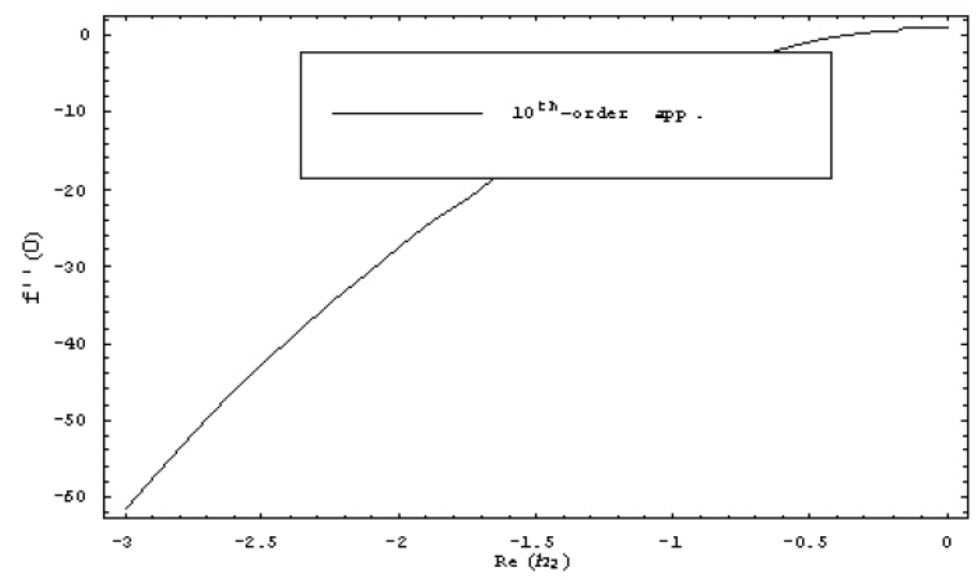

Fig. $5 \hbar_{2}$-curve for discontinuity of derivative at the crest, for the $f(\eta)$ at $\alpha=0.01, \gamma=0.001, c=0.01, \epsilon=0.01, a=1$.

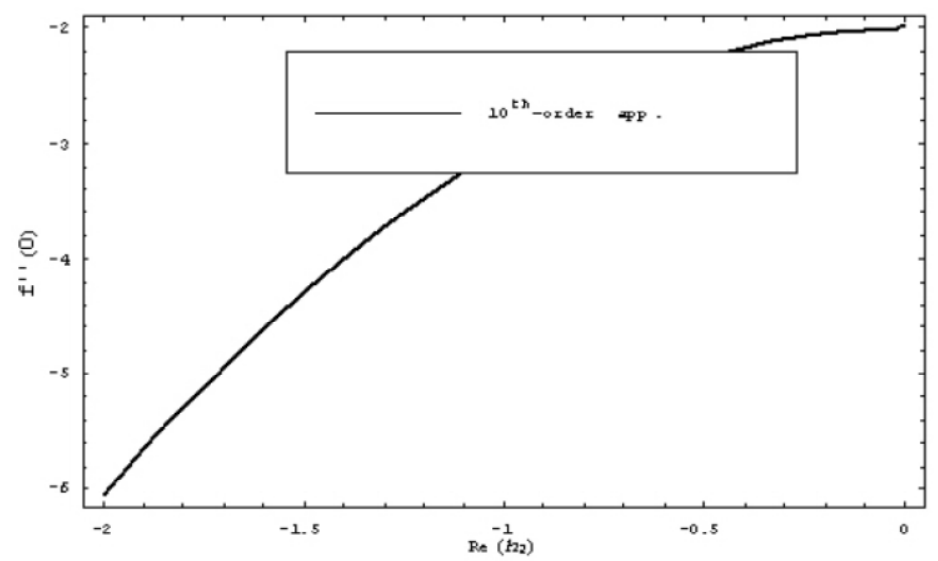

Fig. $6 \hbar_{2}$-curve for continuity of derivative at the crest, for the $f(\eta)$ at $\alpha=0.01, \gamma=0.001, c=0.01, \epsilon=0.01, a=1$. 


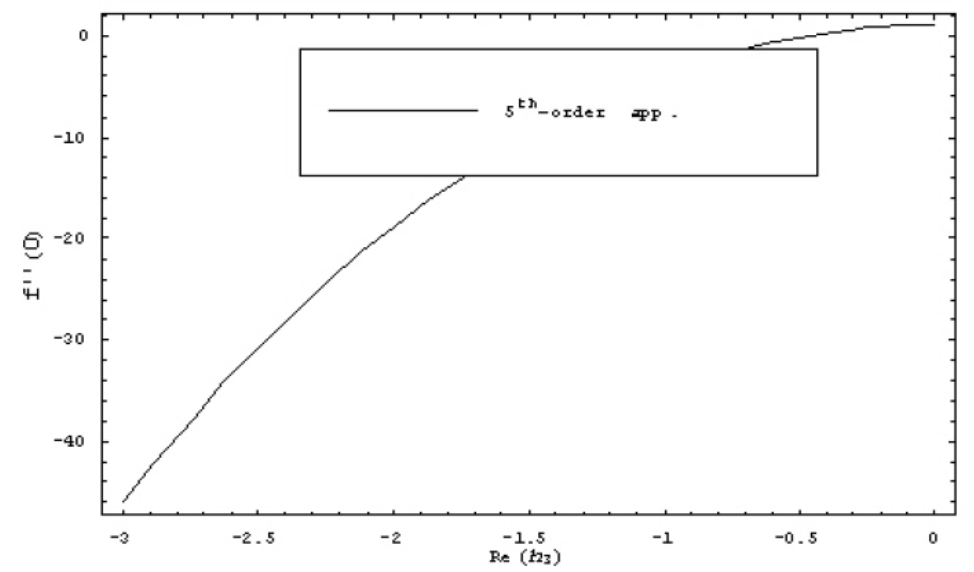

Fig. $7 \hbar_{3}$-curve for discontinuity of derivative at the crest, for the $f(\eta)$ at $\alpha=0.01, c=1, \beta=0.01, a=1, \epsilon=0.01, \delta=0.01$,

$$
\gamma=0.01 \text {. }
$$

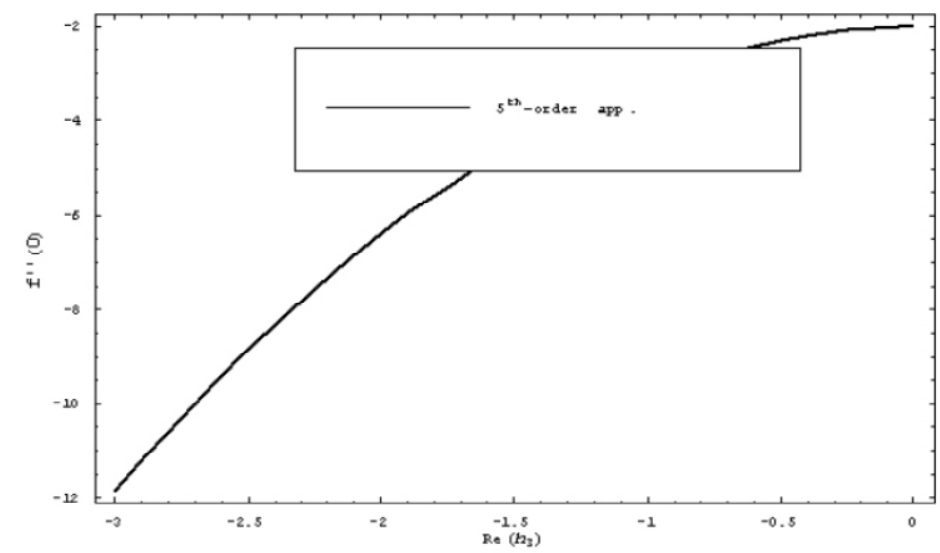

Fig. $8 \hbar_{3}$-curve for continuity of derivative at the crest, for the $f(\eta)$ at $\alpha=0.01, c=1, \beta=0.01, a=1, \epsilon=0.01, \delta=0.01$, $\gamma=0.01$.

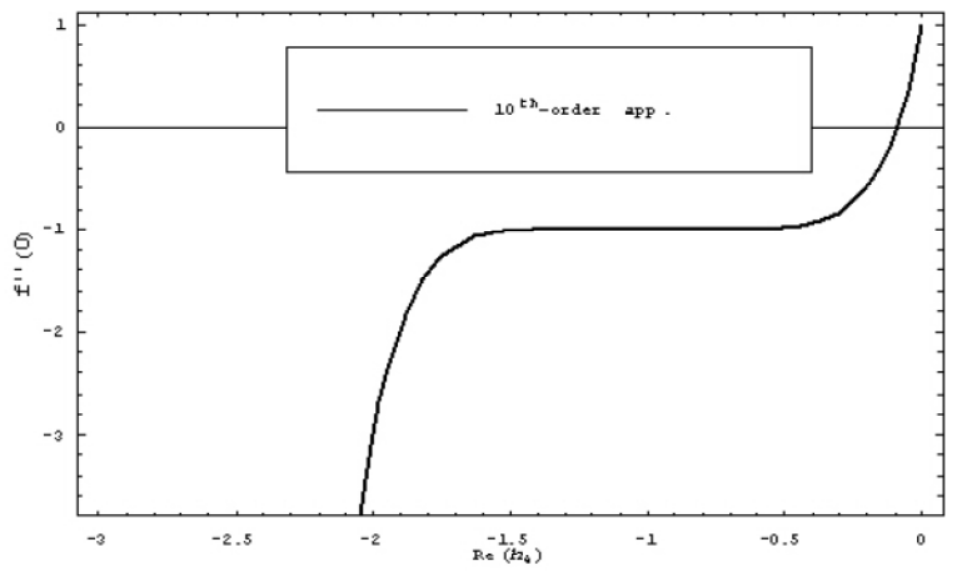

Fig. $9 \hbar_{4}$-curve for discontinuity of derivative at the crest, for the $f(\eta)$ at $c=0.01, a=1$. 


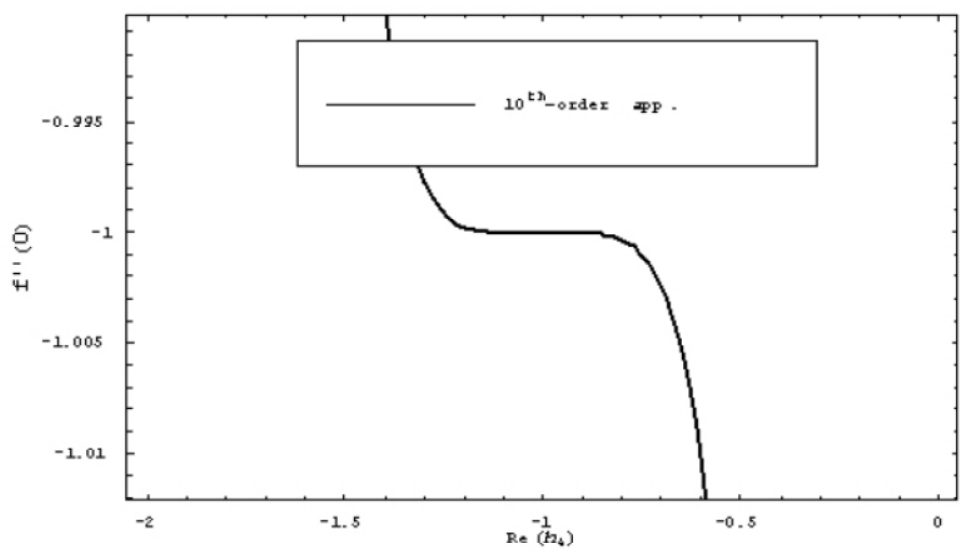

Fig. $10 \hbar_{4}$-curve for continuity of derivative at the crest, for the $f(\eta)$ at $c=0.01, a=1$.

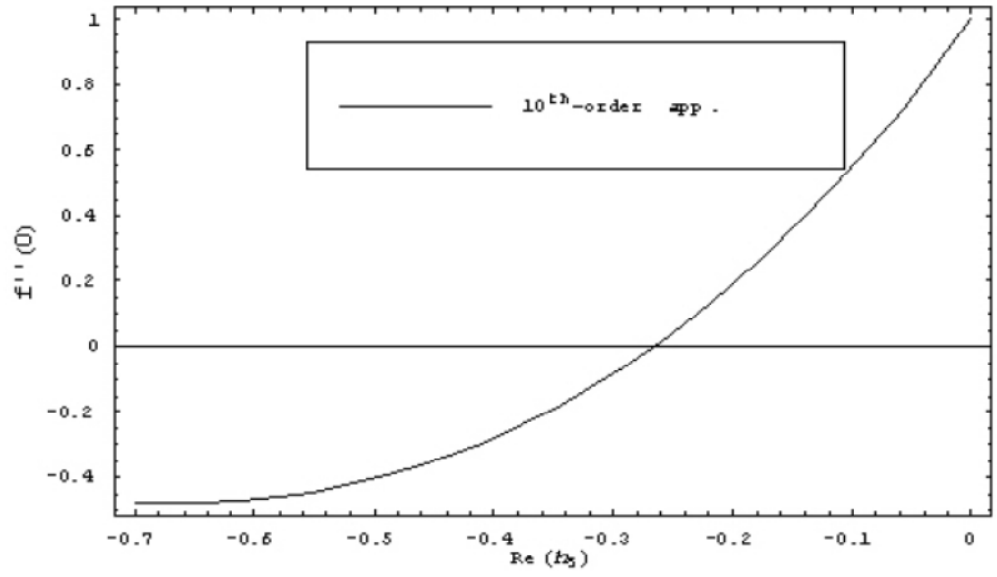

Fig. $11 \hbar_{5}$-curve for discontinuity of derivative at the crest, for the $f(\eta)$ at $\beta_{2}=0.01, \beta_{3}=0.01, \beta_{4}=0.01, \gamma_{1}=0.01, \gamma_{2}=0.01$, $c=1, a=1$.

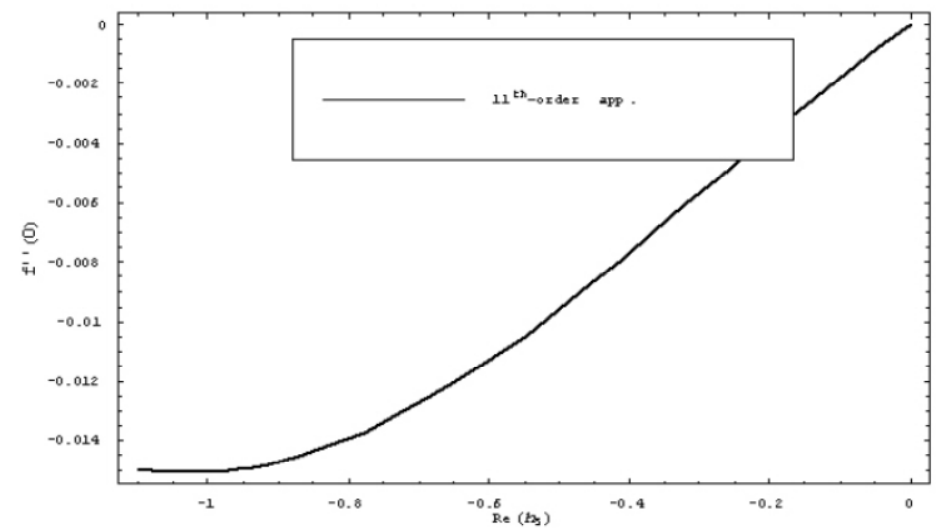

Fig. $12 \hbar_{5}$-curve for continuity of derivative at the crest, for the $f(\eta)$ at $\beta_{2}=0.01, \beta_{3}=0.01, \beta_{4}=0.01, \gamma_{1}=0.01, \gamma_{2}=0.01$, $c=1, a=1$. 


\section{CONCLUSION}

In this paper, solitary waves-series solutions are obtained with and without continuity of the first derivative at crest. The auxiliary linear operator in all the cases is same. The initial guess in all the cases of discontinuity of the derivative at the crest are same and for the cases having continuity of the derivative at the crest are similar. In all the cases series solutions are obtained by taking the values of homotopy parameters $\eta_{1}, \eta_{2}, \eta_{3}, \eta_{4}$ and $\eta_{5}$ from their interval of convergence. The advantage of this method over the other methods is that it itself provides us a convenient way to control the convergence of the approximation series, which shows the flexibility and potential of this method to apply it to nonlinear problems in engineering and science.

\section{ACKNOWLEDGEMENT}

This study was supported by Research University Grant: UKM-GUP-NBT-08-26-095 from, Ministry of Science, Technology and Innovation, Malaysia.

\section{REFERENCES}

[1] Cazenave T, Lions P. "Orbital stability of standing waves for some nonlinear Schrodinger equations." Commun Math Phys 1982; 85: 549-561 Communications in Mathematical Physics 85.4 (1982): 549-61.

[2] Faris WG, Tsay WJ. "Time delay in random scattering." SIAM Journal on Applied Mathematics 54.2 (1994): 443-455.

[3] Bruneau C, Menza L, Lehner T. "Numerical resolution of some nonlinear Schrodinger-like equations in plasmas." Numerical Methods for Partial Differential Equations 15.6 (1999): 672-96.

[4] Abdullaev F, Garnier J. "Solitons in media with random dispersive perturbations." Physica D: Nonlinear Phenomena 134.3 (1999): 303-15.

[5] Debussche A, Menza L. Numerical simulation of focusing stochastic nonlinear Schrodinger equations.. Physica D: Nonlinear Phenomena 162 (2002): 131-54.

[6] Azzouzi F, Triki H, Mezghiche K, Akrmi El A. Solitary wave solutions for high dispersive cubic-quintic nonlinear Schrodinger equation. Chaos Solitons and Fractals 39 (2009): 130407.

[7] Yao LI, Rong Chang JIN. Variational principle for nonlinear Schrodinger equation with high nonlinearity. Journal of Nonlinear Sciences and Applied 1 (2008): 1-4.

[8] Maha Hazmy A El. On the analytical solution of perturbative nonlinear Schrodinger equations with real initial and homogeneous boundary conditions. The Open Applied Mathemathics Journal, 2 (2008): 1-7.

[9] WWang, Mingliang, Xiangzheng Li, and Jinliang Zhang "Various exact solutions of nonlinear Schrodinger equation with two nonlinear terms." Chaos Solitons and Fractals 31.3 (2007): 594-601.

[10] Xu L, Zhang J. "Exact solutions to two higher order nonlinear Schrodinger equations." Chaos Solitons and Fractals 31.3 (2007): 937-42.

[11] SSun, Jian-Qiang, Zhong-Qi Ma, Wei Hua, and Meng-Zhao Qin. New conservation schemes for the nonlinear Schrodinger equation. "Applied Mathematics And Computation 177.1 (2006): 446-51.

[12] Liao SJ. Beyond perturbation: introduction to the homotopy analysis method. Boca Raton: Chapman and Hall/CRC Press; 2003 
[13] Liao SJ. "Notes on the homotopy analysis method: Some definitions and theorems."." Communications in Nonlinear Science and Numerical Simulation14.4 (2009): 983-97.

[14] Liao SJ. "An explicit, totally analytic approximate solution for Blasius' viscous flow problems.”. ." International Journal of Non-Linear Mechanics 34.4 (1999): 759-78.

[15] Liao SJ. "An analytic approximation of the drag coefficient for the viscous flow past a sphere." International Journal of Non-Linear Mechanics 7 (2002): 1-18.

[16] Liao SJ. "An analytic approximate technique for free oscillations of positively damped systems with algebraically decaying amplitude." International Journal of Non-Linear Mechanics 38.8 (2003): 1173-78.

[17] Liao SJ. "An analytic solution of unsteady boundary-layer flows caused by an impulsively stretching plate." Communications in Nonlinear Science and Numerical Simulation 14.4 (2009): 983-97.

[18] Liao SJ, Campo A. "Analytic solutions of the temperature distribution in Blasius viscous flow problems." Journal of Fluid Mechanics 453 (2002): 411-25.

[19] Ayub M, Rashid A, Hayat T. "Exact solution of a third grade fluid past a porous plate using homotopy analysis method." International Journal of English sciences 41.18 (2003):2091103.

[20] AAyub, Muhammad, Haider Zaman, and Masud Ahmad. "Series solution of hydromagnetic flow and heat transfer with Hall effect in a second grade fluid over a stretching sheet." Central European Journal of Physics 8.1 (2010): 135-49.

[21] Ayub M, Zaman H, Sajid M, Hayat T. Analytical solution of stagnation-point flow of a viscoelastic fluid towards a stretching surface, Communications in Nonlinear Science and Numerical Simulation13 (2008): 1822-35.

[22] Hayat T, Khan M, Ayub M. On the explicit analytic solution of an Oldroyd 6-constant fluid. International Journal of English sciences 42 (2004): 123-35.

[23] Hayat T, Khan M, Asghar S. "Homotopy analysis of MHD flows of an Oldroyd 8-constant fluid."." Acta Mechanica 168.3-4 (2004): 213-32.

[24] Hayat T, Khan M, Ayub M. "On non-linear flows with slip boundary condition." " Zeitschrift für angewandte Mathematik und Physik ZAMP 56.6 (2005): 1012-29.

[25] Hayat T, Khan M. "Homotopy solutions for a generalized second-grade fluid past a porous plate." Nonlinear Dynamics 42 (2005): 395-405.

[26] Hayat T, Khan M, Sajid M, Ayub, M. "Steady flow of an Oldroyd 8-constant fluid between coaxial cylinders in a porous medium." Journal of Porous Media 9.8 (2006): 709-22.

[27] Hayat T, Abbas Z, Sajid M, Asghar S. "The influence of thermal radiation on MHD flow of a second grade fluid."International Journal of Heat And Mass Transfer 50 (2007): 931-41.

[28] Hayat T, Sajid M. "Analytic solution for axisymmetric flow and heat transfer of a second grade fluid past a stretching sheet." International Journal of Heat And Mass Transfer 50 (2007): 75-84.

[29] Hayat T, Sajid M. Homotopy analysis of MHD boundary layer flow of an upper-convected Maxwell fluid, International Journal Of english and sciences 45 (2007):343-401.

[30] Hayat T, Zaman H, Ayub M. Analytical solution of hydromagnetic flow with Hall effect over a surface stretching with a power-law velocity. Numerical Methods forPartial Differential Equations.8.1 (2010):135-149.

[31] Abbasbandy S. "The application of homotopy analysis method to nonlinear equations arising in heat transfer." Physics Letters A 360.1 (2006): 109-13. .

[32] Abbasbandy S. Homotopy analysis method for heat radiation equations. International Communications In Heat And Mass Transfer 3493 (2007): 380-87.

[33] Abbasbandy S. The application of homotopy analysis method to solve a generalized HirotaSatsuma coupled KdV equation. Physics Letters A. 361.1 (2007): 478-83.

[34] Ariel PD, Hayat T, Asghar S. Homotopy perturbation method and axisymmetric flow over a stretching sheet, International. Journal. of Nonlinear Science and Numerical Simulation. 7.4 (2006): 399-406. 
[35] Sajid M, Hayat T,Asghar S. "On the analytic solution of the steady flow of a fourth grade fluid." Physics. Letters A.355 (2006):18-26.

[36] Song L, Zhang HQ. Application of homotopy analysis method to fractional KdV-BurgersKuramoto equation, Physics. Letters A 367 (2007):88-94.

[37] ZAyub, Muhammad, Haider Zaman, "Series solution of unsteady free convection flow with mass transfer along an accelerated vertical porous plate with suction." Central European Journal of Physics 8.6 (2010): 931-39.

[38] Ayub, Muhammad, Haider Zaman, and Masud Ahmad Reply to the comments on: Series solution of hydromagnetic flow and heat transfer with Hall effect in a second grade fluid over a stretching sheet." Central European Journal of Physics,8.1 (2010): 135-149. 